\title{
Multiple hydrogen-bonding interactions enhance the solubility of starch in natural deep eutectic solvents: molecule and macroscopic scale insights
}

Chen Cao, Binbin Nian, Yuanfa Liu ${ }^{a *}$

State Key Laboratory of Food Science and Technology, School of Food Science and

Technology, National Engineering Research Center for Functional Food, National Engineering Laboratory for Cereal Fermentation Technology, Collaborative Innovation Center of Food Safety and Quality Control in Jiangsu Province, Jiangnan University, 1800 Lihu Road, Wuxi 214122, Jiangsu, People's Republic of China Corresponding Authors: Yuanfa Liu

* Tel.: +860510-85876799, Fax: +860510-85876799 E-mail: yfliu@jiangnan.edu.cn 
Number of pages: \#7

Number of figures: \#2

Number of tables: \#2 


\section{Abbreviations}

\begin{tabular}{cc}
\hline Abbreviations & Full name \\
\hline NADESs & Natural deep eutectic solvents \\
G50 & Gelose starch \\
XRD & X-ray diffraction analysis \\
ATR-FTIR & Attenuated total reflection Fourier transform infrared spectroscopy \\
MD & Molecular dynamics study \\
HBA & Hydrogen-bonding acceptors \\
HBD & Hydrogen-bonding donors \\
ILs & Ionic liquids \\
\hline
\end{tabular}



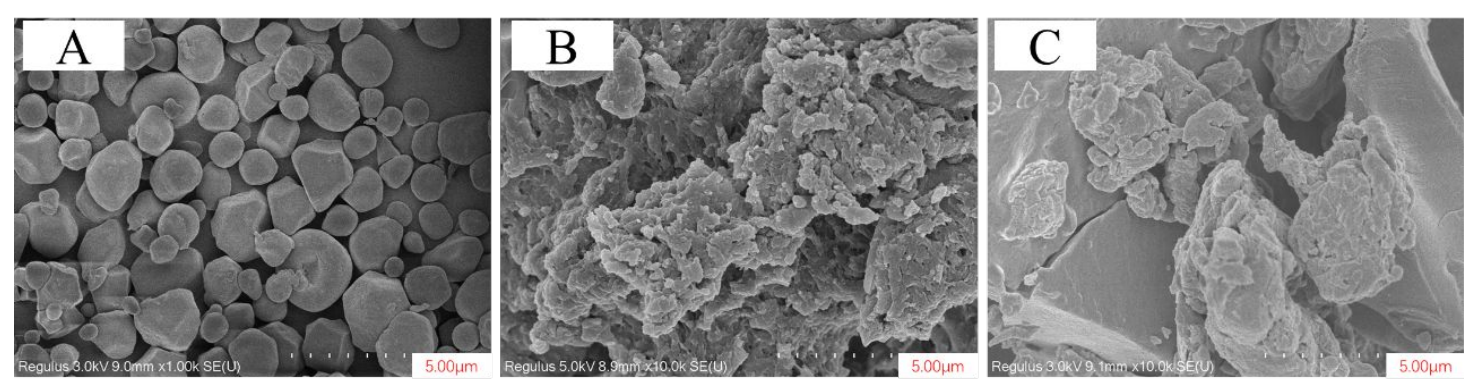

Figure S1. Scanning electron microscope (SEM) of G50 (A) and regenerated G50 from

BU (B) and MGlu (C) 


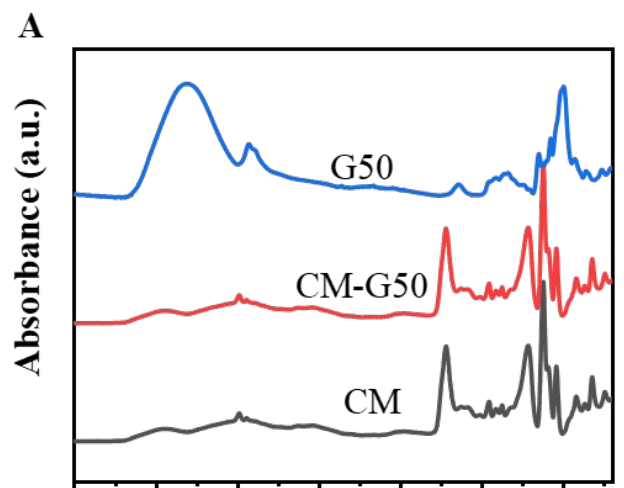

4000350030002500200015001000

Wavenumber $\left(\mathrm{cm}^{-1}\right)$

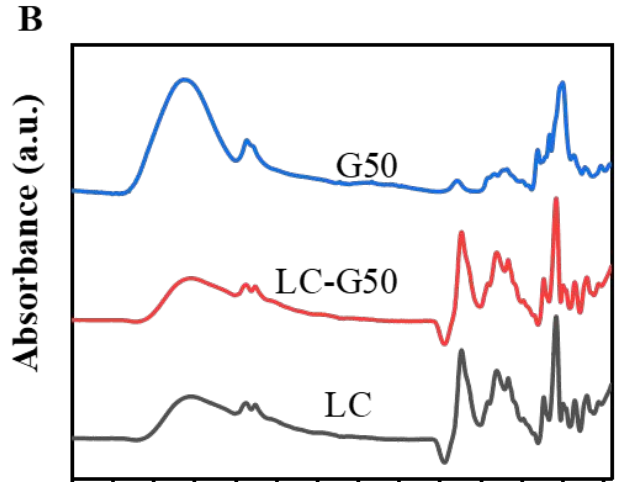

4000350030002500200015001000

Wavenumber $\left(\mathrm{cm}^{-1}\right)$

Figure S2. ATR-FTIR spectra for G50 dispersed in CM (A) and LC (C) 
Table S1. The possibility NADESs in which starch may have a high solubility

\begin{tabular}{ccccc}
\hline S. no. & HBA & HBD & HBA : HBD & Fluidity at 363K \\
\hline 1 & Betaine & xylitol & $1: 2$ & yes $^{\mathrm{a}}$ \\
2 & Betaine & Malic acid & $1: 2$ & no \\
3 & Betaine & Citric acid & $1: 1$ & no \\
4 & Betaine & Glycerin & $1: 2$ & yes \\
5 & Betaine & Urea & $1: 2$ & yes \\
6 & Choline chloride & xylitol & $1: 2$ & yes \\
7 & Choline chloride & Malic acid & $1: 1$ & yes \\
8 & Choline chloride & glucose & $3: 2$ & no \\
9 & Choline chloride & Trehalose & $1: 3$ & no \\
10 & Choline chloride & Glycerin & $1: 2$ & yes \\
11 & Choline chloride & Urea & $1: 1$ & yes \\
12 & Choline chloride & Lactic acid & $1: 10$ & yes \\
13 & Proline & Sorbitol & $1: 1$ & no \\
14 & Proline & Glycerin & $1: 2$ & yes \\
15 & Proline & Urea & $1: 2$ & no \\
16 & Lactic acid & Glycine & $1: 3$ & yes \\
17 & Malic acid & Glucose & $1: 3$ & yes \\
18 & Malic acid & Sucrose & $1: 3$ & no \\
19 & Malic acid & Glycerin & $1: 1$ & yes \\
$*$ HBA:HBD are given in molar ratios; yes ${ }^{\mathrm{a}}$ was liquid, but high viscous & \\
\hline
\end{tabular}


Table S2. Second screening round of NADES

\begin{tabular}{ccccc}
\hline S. no. & HBA & HBD & HBA : HBD & Fluidity at 363 K \\
\hline 1 & Betaine & xylitol & $1: 01$ & yes $^{\mathrm{a}}$ \\
2 & Betaine & xylitol & $2: 01$ & NA \\
3 & Betaine & Urea & $1: 1$ & yes \\
4 & Betaine & Urea & $2: 1$ & no \\
5 & Choline chloride & xylitol & $1: 1.5$ & yes \\
6 & Choline chloride & xylitol & $1: 01$ & no \\
7 & Choline chloride & Trehalose & $3: 01$ & yes \\
8 & Choline chloride & Trehalose & $1: 01$ & no \\
9 & Choline chloride & Urea & $1: 02$ & yes \\
10 & Choline chloride & Urea & $1: 1.5$ & no \\
11 & Lactic acid & Glycine & $1: 01$ & NA \\
12 & Lactic acid & Glycine & $3: 01$ & NA \\
13 & Lactic acid & Glycine & $6: 01$ & NA \\
14 & Lactic acid & Glycine & $9: 01$ & yes ${ }^{\mathrm{a}}$ \\
15 & Malic acid & Glucose & $1: 02$ & NA \\
16 & Malic acid & Glucose & $1: 01$ & yes \\
$*$ HBA:HBD are given in molar ratios; yes ${ }^{\mathrm{a}}$ was liquid, but high viscous; NA represents that NADESs cannot be \\
formed
\end{tabular}

